\title{
The clinical characteristics of retinal vasculitis in HLA-B27-positive patients
}

\section{Arthur Menno Braakenburg \& Aniki Rothova}

To cite this article: Arthur Menno Braakenburg \& Aniki Rothova (2014) The clinical characteristics of retinal vasculitis in HLA-B27-positive patients, Ocular Immunology and Inflammation, 22:3, 197-202, DOI: 10.3109/09273948.2013.835429

To link to this article: http://dx.doi.org/10.3109/09273948.2013.835429

Published online: 08 Oct 2013.

Submit your article to this journal $\sqsubset$

Џlll Article views: 147

Q View related articles $\sqsubset$

View Crossmark data ¿ 


\title{
The clinical characteristics of retinal vasculitis in HLA-B27-positive patients*
}

\author{
Arthur Menno Braakenburg ${ }^{1}$ and Aniki Rothova ${ }^{2}$ \\ ${ }^{1}$ Department of Ophthalmology, VU University Medical Center Amsterdam, Amsterdam, Netherlands and \\ ${ }^{2}$ Department of Ophthalmology, Erasmus Medical Center Rotterdam, Rotterdam, Netherlands
}

\begin{abstract}
Purpose: To investigate the ocular and systemic manifestations of retinal vasculitis in HLA-B27-positive patients. Methods: Retrospective noncomparative case series of 9 HLA-B27-positive patients with uveitis and retinal vasculitis. Main outcome measures consisted of ocular and angiographic findings and assessment of any additional systemic disorders.

Results: Three male and 6 female HLA-B27-positive patients with a median age of 32 years were diagnosed with retinal vasculitis. Concurrent intraocular inflammation was noted in all patients. All patients suffered from extensive vasculitis of the large retinal veins. Five patients developed retinal vasculitis at the onset of uveitis and the remaining 4 exhibited retinal vasculitis 1-15 years after the onset of uveitis. Vascular occlusions occurred in 4 patients and subsequent neovascularizations developed in 3. Three patients were diagnosed with an HLA-B27-associated systemic disease.

Conclusion: Retinal vasculitis may develop in the wake of HLA-B27-associated uveitis and might represent a rare manifestation of HLA-B27-associated disease.
\end{abstract}

Keywords: retinal, vasculitis, HLA-B27, ankylosing, spondylitis

HLA-B27-associated uveitis typically presents as a recurrent unilateral acute anterior uveitis (AAU), ${ }_{1}^{1}$ although posterior segment manifestations in HLA-B27-associated uveitis are regularly reported. ${ }^{2-4}$ The most common posterior segment manifestations include vitritis, cystoid macular edema (CME), and optic disc leakage. ${ }^{2-4}$ Occasional cases of retinal vasculitis in HLA-B27-positive patients are also documented, and constitute about $20-25 \%$ of those with posterior segment involvement. ${ }^{2,4-9}$ It is not known whether the association of HLA-B27-antigen and retinal vasculitis is coincidental or whether retinal vasculitis might occur as a specific manifestation of an HLA-B27-associated disease.

The aim of this study is to report on 9 HLAB27-positive patients with retinal vasculitis and describe the clinical characteristics, fluorescein angiography (FA) findings, and visual outcomes.

\section{MATERIAL AND METHODS}

In this study we included 9 patients with retinal vasculitis who were HLA-B27-positive. Five patients were identified in the cohort of 177 HLA-B27-positive patients presenting with typical AAU, sometimes alternating, at the University Medical Center Utrecht between 1995 and 2005. This data set included $10(6 \%)$ patients with suspected retinal vasculitis based on the basis of the presence of perivascular sheathing and/or cuffing; however, fluorescein angiographies met our inclusion criteria in only in 5 (3\%) patients. The remaining 4 patients were

*This study was approved by the METC (Medical Ethical Testing Committee) of the University Medical Center in Utrecht. Received 5 June 2013; revised 4 August 2013; accepted 13 August 2013; published online 8 October 2013

Correspondence: Arthur Menno Braakenburg, Department of Ophthalmology, VU University Medical Center Amsterdam, Amsterdam, Netherlands, Email: a.braakenburg@vumc.nl 
selected from our database file of patients with retinal vasculitis.

All patients underwent a standard screening consisting of erythrocyte sedimentation rate, red and white blood cell counts, determination of serum angiotensin-converting enzyme levels, serologic testing for syphilis and borreliosis, chest radiography, and antinuclear antibody testing (ANA). In addition, all patients were examined by a specialist in internal medicine to assess the possible presence of associated systemic disease. We identified 1 additional HLA-B27positive patient with retinal vasculitis who was diagnosed with ankylosing spondylitis (AS) and Crohn disease; however, this patient was excluded from the present series since this association was previously noted. ${ }^{10,11}$ Also, 1 HLA-B27-positive patient with retinal vasculitis who also had a biopsy-proven pulmonary sarcoidosis was excluded from this our study.

In general, patients with retinal vasculitis are only rarely typed for HLA-B27. In our center, only patients with anterior uveitis and panuveitis are systematically typed for HLA-B27, whereas patients with retinal vasculitis who present mostly with posterior uveitis are generally not typed for HLA-B27.

At present, there is no consensus on the definition of retinal vasculitis. ${ }^{12}$ Therefore, for the purpose of this study we adopted the following definition: moderate to severe leakage of the large retinal vessels during the middle and late FA frames and/or occlusive retinal vasculopathy in the setting of ocular inflammation. In the present study, the presence of optic disc leakage, CME and slight leakage, and/or late staining of the small retinal vessels (capillaries) was in this study not considered to indicate genuine retinal vasculitis, and, consequently, these patients were not included. Also, slight and local perivascular sheathing/cuffing alone was not considered to be retinal vasculitis. The extension of vasculitis was classified according to its location in 5 parts of the retina, specifically, the posterior pole and four peripheral quadrants. All fluorescein angiograms were evaluated and coded by the 2 independent ophthalmologists; in the case of whenever there were differing evaluations, the angiograms were discussed until an agreement on the diagnosis was reached.

Multiple variables were assessed, such as age at onset of ocular disease, systemic and ocular medical history, and various clinical manifestations, including severity, complications, treatment, and visual outcomes. Systemic HLA-B27-associated diseases were diagnosed according to standard diagnostic criteria. ${ }^{13}$

\section{RESULTS}

Information on the general data of 9 HLAB27-positive patients and their associated systemic diseases is given in Table 1. The median age at onset of retinal vasculitis was 32 years (range 17-52 years). The median follow-up time from the onset of retinal vasculitis was 5 years (range 6 months to 8 years). Three patients were diagnosed with an HLAB27-associated systemic disease: 1 with AS, 1 with a combination of juvenile idiopathic arthritis (JIA) and psoriasis, and 1 with an undifferentiated spondylarthropathy. One additional patient was diagnosed with palindromic rheumatism (Table 1). All of these systemic disorders were manifest before the onset of retinal vasculitis.

Retinal vasculitis did not occur as an isolated phenomenon in any of our patients, but it always developed with concurrent anterior or panuveitis. Five patients had retinal vasculitis from the onset of uveitis and in 4 of them it was preceded by a typical

TABLE 1. General characteristics of 9 human leukocyte antigen (HLA)-B27-positive patients with retinal vasculitis.

\begin{tabular}{|c|c|c|c|c|c|}
\hline Patient no. & Gender & $\begin{array}{l}\text { Age at onset of } \\
\text { uveitis (years) }\end{array}$ & $\begin{array}{l}\text { Age at onset } \\
\text { of RV (years) }\end{array}$ & Systemic disease & Treatment of RV \\
\hline 1 & M & 51 & 52 & AS & $\begin{array}{l}\text { Systemic and periocular CS, } \\
\text { MTX, photocoagulation }\end{array}$ \\
\hline 2 & M & 20 & 20 & None & $\begin{array}{l}\text { Periocular and systemic CS, } \\
\text { PPV, photocoagulation, AZA }\end{array}$ \\
\hline 3 & $\mathrm{~F}$ & 33 & 33 & None & $\begin{array}{l}\text { Periocular and systemic CS, } \\
\text { PPV }\end{array}$ \\
\hline 4 & M & 31 & 32 & USPA & Periocular CS \\
\hline 5 & $\mathrm{~F}$ & 28 & 28 & None & Periocular CS \\
\hline 6 & $\mathrm{~F}$ & 17 & 17 & None & Systemic CS \\
\hline 7 & $\mathrm{~F}$ & 11 & 26 & JIA, psoriasis & $\begin{array}{l}\text { Systemic and periocular CS, } \\
\text { AZA, CsA }\end{array}$ \\
\hline 8 & $\mathrm{~F}$ & 28 & 35 & Palindromic rheumatism & $\begin{array}{l}\text { Systemic and periocular CS, } \\
\text { MTX, photocoagulation }\end{array}$ \\
\hline 9 & $\mathrm{~F}$ & 38 & 38 & None & $\begin{array}{l}\text { Periocular CS, photocoagula- } \\
\text { tion, PPV }\end{array}$ \\
\hline
\end{tabular}

Note. M, male; F, female; AS, ankylosing spondylitis; AZA, azathioprine; CS, corticosteroids; CsA, cyclosporine A; MTX, methotrexate; PPV, pars plana vitrectomy; RV, retinal vasculitis; USPA, undifferentiated spondylarthritis; JIA, juvenile idiopathic arthritis. 
acute anterior uveitis after which the retinal vasculitis developed several years later (Table 1). Eight of our 9 patients (89\%) had bilateral retinal vasculitis (in total 17 affected eyes; Table 2).

In all but 1 patient (patient 2, Table 1) the duration of uveitis and retinal vasculitis was longer than 3 months. The exact duration of the retinal vasculitis activity was difficult to assess, but in at least of the 9 patients active retinal vasculitis persisted even when the uveitis became quiescent. Involvement of the large veins was noted in all patients, whereas retinal arteritis was not seen in any of the patients (Figure 1). Peripheral retinal quadrants were involved in all affected eyes, and in 5 patients $(10 / 17 ; 59 \%$ of the affected eyes) the vessels of the posterior pole were also affected. In 5 patients $(8 / 17 ; 47 \%$ of the affected eyes) the retinal vasculitis was accompanied by vascular occlusions and in 6 patients $(10 / 17 ; 53 \%$ of the affected eyes) by retinal ischemia. In 3 patients (6/17; $35 \%$ of the affected eyes) neovascularizations developed. Retinal hemorrhages were present in 6 patients $(8 / 17 ; 47 \%$ of the affected eyes). Seven patients $(13 / 17 ; 76 \%$ of the affected eyes) developed $\mathrm{CME}$, and 7 patients $(12 / 17 ; 71 \%$ of the affected eyes) had optic disc leakage. Vitreous hemorrhages developed in 3 patients, 2 of whom them underwent a pars plana vitrectomy (PPV). All vitreous hemorrhages were in eyes with neovascularizations.

TABLE 2. Clinical characteristics of 9 human leukocyte antigen (HLA-B27)-positive patients with retinal vasculitis.

\begin{tabular}{|c|c|c|c|c|c|c|c|c|c|c|}
\hline \multirow{2}{*}{$\begin{array}{l}\text { Patient } \\
\text { no. }\end{array}$} & \multirow[b]{2}{*}{ Eye } & \multirow[b]{2}{*}{ RV } & \multirow{2}{*}{$\begin{array}{c}\text { Location of RV } \\
\text { (no. of affected } \\
\text { quadrants) }\end{array}$} & \multirow{2}{*}{$\begin{array}{c}\text { Optic disc } \\
\text { leakage }\end{array}$} & \multirow[b]{2}{*}{ CME } & \multirow{2}{*}{$\begin{array}{l}\text { Retinal } \\
\text { ischemia }\end{array}$} & \multirow[b]{2}{*}{ Neovascularization } & \multicolumn{3}{|c|}{ Visual acuity } \\
\hline & & & & & & & & Onset RV & 6-month FU & 3-year FU \\
\hline \multirow[t]{2}{*}{1} & Right & Yes & $\mathrm{PP}$, peripheral (4) & No & Yes & Yes & Yes & 0.4 & 1.0 & 1.0 \\
\hline & Left & Yes & $\mathrm{PP}$, peripheral (4) & No & Yes & Yes & Yes & 0.6 & 1.0 & 0.6 \\
\hline \multirow[t]{2}{*}{2} & Right & Yes & $\mathrm{PP}$, peripheral (2) & Yes & Yes & Yes & Yes & 0.05 & 1.0 & 1.0 \\
\hline & Left & Yes & $\mathrm{PP}$, peripheral (1) & Yes & Yes & Yes & Yes & 1.2 & 1.0 & 1.0 \\
\hline \multirow[t]{2}{*}{3} & Right & Yes & Peripheral (4) & Yes & Yes & Yes & No & 0.8 & 0.8 & 1.0 \\
\hline & Left & Yes & Peripheral (3) & Yes & Yes & Yes & No & 0.5 & 0.8 & 0.8 \\
\hline \multirow[t]{2}{*}{4} & Right & Yes & Peripheral (2) & Yes & Yes & No & No & 1.2 & 1.0 & 1.0 \\
\hline & Left & Yes & Peripheral (2) & Yes & Yes & No & No & 1.0 & 1.0 & 1.0 \\
\hline \multirow[t]{2}{*}{5} & Right & No & NA & NA & NA & NA & NA & NA & NA & NA \\
\hline & Left & Yes & Peripheral (2) & Yes & Yes & Yes & No & 0.16 & 0.8 & NA \\
\hline \multirow[t]{2}{*}{6} & Right & Yes & $\mathrm{PP}$, peripheral (3) & Yes & No & No & No & 0.63 & 1.0 & 1.0 \\
\hline & Left & Yes & $\mathrm{PP}$, peripheral (2) & Yes & No & No & No & 0.8 & 1.0 & 1.0 \\
\hline \multirow[t]{2}{*}{7} & Right & Yes & $\mathrm{PP}$, peripheral (2) & Yes & Yes & No & No & 0.3 & 0.4 & 1.0 \\
\hline & Left & Yes & $\mathrm{PP}$, peripheral (4) & Yes & Yes & No & No & 0.4 & 0.6 & 0.7 \\
\hline \multirow[t]{2}{*}{8} & Right & Yes & $\mathrm{PP}$, peripheral (2) & Yes & Yes & Yes & No & 0.2 & $0.5^{\mathrm{a}}$ & NA \\
\hline & Left & Yes & $\mathrm{PP}$, peripheral (2) & No & Yes & No & No & 1.0 & $0.3^{\mathrm{a}}$ & NA \\
\hline \multirow[t]{2}{*}{9} & Right & Yes & Peripheral (3) & No & No & Yes & Yes & 1.0 & 1.0 & 1.0 \\
\hline & Left & Yes & Peripheral (3) & No & No & Yes & Yes & 0.5 & 0.5 & $0.5^{\mathrm{b}}$ \\
\hline Total & & $17(94 \%)$ & & $12(71 \%)$ & $13(76 \%)$ & $10(59 \%)$ & $6(35 \%)$ & & & \\
\hline
\end{tabular}

Note. CME, cystoid macular edema; FU, follow-up; NA, not applicable; PP, posterior pole; RV, retinal vasculitis.

${ }^{\text {a }}$ Loss of vision caused by CME.

${ }^{\mathrm{b}}$ Temporary low vision caused by cataract.

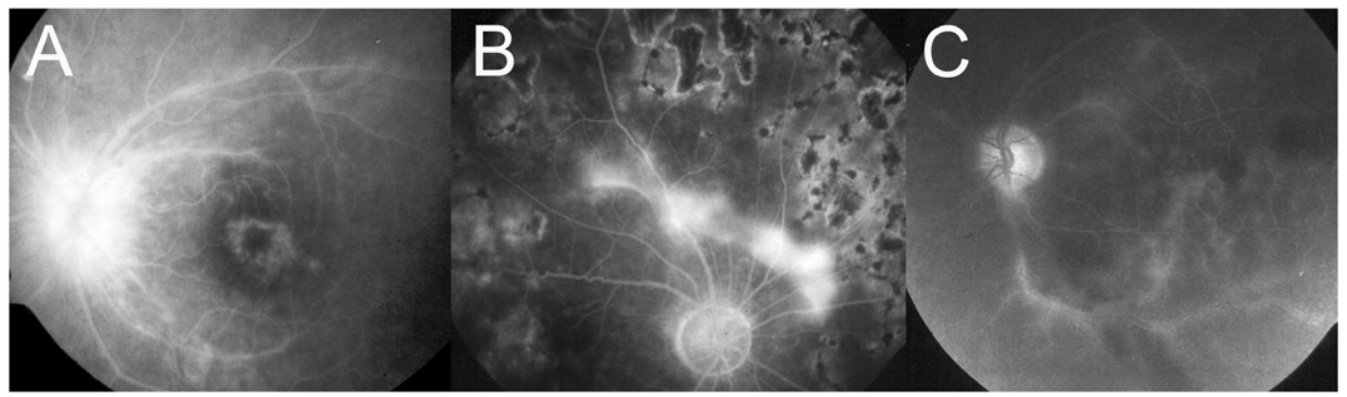

FIGURE 1. (A) Retinal vasculitis, optic disc leakage and cystoid macular edema in a 26-year-old HLA-B27-positive female patient with recurrent attacks of anterior uveitis from 11 years of age, juvenile idiopathic arthritis and psoriasis (Table 1 and 2, patient no. 7). (B) Mild optic disc leakage and not yet regressed neovascular membrane above the optic disc following the partial panlaser photocoagulation and treatment with systemic steroids and methotrexate in a 52-year-old HLA-B27-positive patient with ankylosing spondylitis and previous attacks of acute anterior uveitis (Table 1 and 2, patient no. 1). Note the irregular vascular walls and the residual staining of the veins in the perimacular region. (C) The opposite eye of the same patient (no. 1) exhibiting leakage of the big retinal veins on the late frames of fluorescein angiography. 
Treatment of retinal vasculitis is shown in Table 1. In 5 patients, (including 1 who also received peripheral photocoagulation), the uveitis and retinal vasculitis reacted well to systemic and/or periocular steroids. In 4 patients the retinal vasculitis and/or uveitis became steroid-resistant and additional immunosuppressive medications, such as cyclosporine A, methotrexate (MTX), and/or azathioprine, were required. Of these 4 patients, 1 was diagnosed with AS, 1 with a combination of JIA and psoriasis, 1 with palindromic rheumatism, and in 1 patient no systemic disease was found. The JIA patient suffered from arthritis and, initially, from alternating unilateral AAU attacks; she was negative for ANA, but positive for HLA-B27. Four patients received photocoagulation and 3 underwent PPV.

At the onset of retinal vasculitis, 5 patients (6/17; $35 \%$ affected eyes) had a visual acuity of less than 0.5 . At 6 months follow-up, 2 patients had with a visual acuity of less than 0.5, both unilateral. At 3-year follow-up, none of the 7 followed patients had a visual acuity of less than 0.5 .

\section{SELECTED CASE: PATIENT I}

A 52-year-old HLA-B27-positive male was referred to our institute with retinal vasculitis in the right eye, which developed approximately 1 year after recurrent attacks of alternating AAU, which had now progressed to panuveitis and development of retinal vasculitis. Medical history revealed that he developed AS at age 27 years. Three months after the onset of vasculitis in the right eye, retinal vasculitis progressed to bilateral involvement. FA revealed a retinal vasculitis of the central and peripheral veins associated with retinal hemorrhages, vascular occlusions, and development of neovascularizations, optic disc leakage, and CME (Figure 1). Subsequently, the patient developed recurrent vitreous hemorrhages in the right eye. In addition to local treatment, the patient received periocular steroid injections, systemic steroids (initial dose $60 \mathrm{mg}$ ), and MTX (initial dose $10 \mathrm{mg} /$ week) with the addition of folic acid. Inflammation of the anterior segment and the vitreous subsided within 2 months. In the following months, panretinal photocoagulation was performed in both eyes. After this treatment, FA revealed a mild activity of retinal vasculitis in both eyes with optic disc leakage and decreased neovascularizations. The uveitis in both eyes was quiescent and the systemic corticosteroids were gradually reduced. Three years later the patient developed a recurrence of active bilateral occlusive retinal vasculitis complicated by $\mathrm{CME}$, despite the ongoing treatment with MTX. Periocular steroids were added and retinal vasculitis activity slowly decreased. Four years after the onset of retinal vasculitis the patient is still on MTX and his visual acuity is 1.0 in the right eye and 0.6 in the left eye.

\section{DISCUSSION}

In a cohort of 177 HLA-B27-positive patients initially presenting with anterior uveitis, we identified retinal vasculitis in $5(3 \%)$ patients and identified an additional 4 more HLA-B27-positive patients among those the patients with retinal vasculitis. All HLAB27-positive retinal vasculitis patients had concurrent panuveitis and 4 had previous AAU attacks typical for HLA-B27-associated uveitis. The most common FA findings included extensive phlebitis, which was in most cases associated with optic disc leakage and CME. Although 4 patients became steroidresistant and 3 developed neovascularizations with vitreous hemorrhages, in all followed patients the visual acuity at the 3-year follow-up was 0.5 or better.

The association between retinal vasculitis and HLA-B27 might be a coincidence, but might also represent a true, but rare association where retinal vasculitis forms a part of an HLA-B27-associated disorder. In our series, 4 of the patients had previous attacks of AAU, typical for HLA-B27-associated uveitis, and 3 patients (patients 1, 4 and 7 in Table 1) suffered from systemic diseases known to be linked to the HLA-B27 antigen. ${ }^{1}$ No additional causes or explanations for retinal vasculitis were found in our patients. These findings support the hypothesis that retinal vasculitis might occur in the wake of an HLA-B27-associated disorder. Several reports on retinal vasculitis in HLA-B27-positive patients were previously published and were considered a part of atypical posterior segment manifestations in HLA-B27-associated uveitis. ${ }^{24-9}$ From a cohort of 166 patients with HLA-B27-associated uveitis, Rodriguez et al. reported 7 (4\%) patients with retinal vasculitis, which is in accordance with our findings. ${ }^{4}$ The occurrence of retinal vasculitis in HLA-B27-associated disease may in fact even be higher than 3\%, as not all our patients with retinal vasculitis were tested for HLA-B27. Furthermore, if all patients with HLA-B27-associated uveitis underwent FA the percentage might be higher, as retinal vasculitis can be missed if based on fundoscopic examination alone. However, approximately $8 \%$ of the people in Western Europe are HLA-B27-positive ${ }^{14}$ and certainly in some cases the association between retinal vasculitis and the HLA-B27 antigen might be coincidental.

Multiple reports were published on retinal vasculitis occurring in Crohn disease, where retinal vasculitis probably represents a rare complication of this systemic disorder. ${ }^{10,11}$ One of our retinal 
vasculitis patients was diagnosed with both Crohn disease and AS.

An interesting question is whether retinal vasculitis in HLA-B27-positive patients is confined to the eyes or might merely represent merely a symptom of a generalized vasculitis. There are multiple reports of aortic arteritis, polyarteritis nodosa, and Takayasu arteritis in HLA-B27-positive individuals or in patients with an already established HLAB27-associated disorder. ${ }^{15-17}$ In our patients, no overt signs of systemic vasculitis were noted. Future research might include a systematic search for systemic vasculitis in affected patients.

Our cohort shared many of the clinical characteristics with the HLA-B27-positive AAU population, specifically the young age at onset, associated systemic diseases, and a favorable visual prognosis. ${ }^{1}$ The only exception was a slight female preponderance in contrast to the HLA-B27-associated AAU population. ${ }^{1}$ It is possible that females with HLAB27-associated uveitis are more prone to atypical ocular presentations, similar to other HLA-B27-associated systemic disorders, such as AS. ${ }^{3,6,18,19}$ Although HLA-B27-associated uveitis is generally of the acute type located in the anterior eye segment, the uveitis associated with retinal vasculitis was always panuveitis with generally a duration longer than 3 months.

Of our 9 HLA-B27-positive patients with retinal vasculitis, $3(33 \%)$ HLA-B27-positive patients with retinal vasculitis developed neovascularizations, which is higher than the approximately $15 \%$ found in other retinal vasculitis types. ${ }^{8,20}$ The majority of our patients with retinal vasculitis developed CME (63\%), which is consistent with previous findings in idiopathic retinal and retinal vasculitis in Behçet patients. ${ }^{8}$

Until now, less than 35 HLA-B27-positive patients with retinal vasculitis have been reported. ${ }^{2,4-9}$ However, these patients were not thoroughly studied and their clinical and FA characteristics were not well described. Although Yalçindag et al. published a case report on occlusive retinal vasculitis in an HLAB27-positive patient, in other cohorts no retinal vascular occlusions or the development of neovascularizations were found in their cohort of patients. ${ }^{2,8,9}$ In the present study, a problem during follow-up was the assessment of the duration of retinal vasculitis activity. FA was not regularly performed and the fundoscopy was not always reliable. Of our 9 patients, 3 FA demonstrated extended retinal vasculitis, which was not suspected on fundoscopic findings.

In general, the visual outcome of retinal vasculitis depends on the underlying cause, the presence of ischemia, and the treatment given. Despite the relatively high number of complications, such as retinal ischemia and the development of neovascularizations, in our series the visual prognosis was favorable as none of the 7 patients who completed 3-year follow-up had developed visual acuity of less than 0.5; however, we did lose 2 patients during the follow-up period.

Our study has all the shortcomings of a retrospective study and lacks comparison with retinal vasculitis in HLA-B27-negative patients. None of the patients had indications of any other causes of retinal vasculitis, although these might develop in the future. In addition, we cannot report on the exact prevalence of vasculitis in HLA-B27-associated uveitis. To answer the question of whether retinal vasculitis is more prevalent in HLA-B27-positive individuals compared to their HLA-B27-negative counterparts would require a large prospective study in which all patients presenting with retinal vasculitis are were typed for HLA-B27.

In conclusion, our study shows that, in this small cohort, the finding of retinal vasculitis with panuveitis may occur in the wake of HLA-B27-associated disorders and may represent an infrequent manifestation of HLA-B27-associated ocular disease.

\section{DECLARATION OF INTEREST}

The authors report no conflicts of interest. The authors alone are responsible for the content and writing of the paper.

\section{REFERENCES}

1. Chang JH, McCluskey PJ, Wakefield D. Acute anterior uveitis and HLA-B27. Surv Ophthalmol. 2005;50:364-388.

2. Kim SJ, Chung H, Yu HG. Posterior segment involvement in Korean patients with HLA-B27-associated uveitis. O Immunol Inflamm. 2009;17:26-32.

3. Monnet D, Breban M, Hudry C, et al. Ophthalmic findings and frequency of extraocular manifestations in patients with HLA-B27 uveitis: a study of 175 cases. Ophthalmology. 2004;111:802-809.

4. Rodriguez A, Akova YA, Pedroza-Seres M, et al. Posterior segment ocular manifestations in patients with HLAB27-associated uveitis. Ophthalmology. 1994;101:1267-1274.

5. Ahn JK, Park YG. Human leukocyte antigen B27 and B51 double-positive Behçet uveitis. Arch Ophthalmol. 2007;125: 1375-1380.

6. Braakenburg AM, de Valk HW, de Boer JH, et al. Human leukocyte antigen-B27-associated uveitis: long-term follow-up and gender differences. Am J Ophthalmol. 2008; 145:472-479.

7. Galluzzo E, Taglione E, Bartolomei MP, et al. Coexistence of ankylosing spondylitis and undifferentiated connective tissue disease. Clin Rheumatol. 1998;17:236-238.

8. Graham EM, Stanford MR, Sanders MD, et al. A point prevalence study of 150 patients with idiopathic retinal vasculitis, 1: diagnostic value of ophthalmological features. Br J Ophthalmol. 1989;73:714-721.

9. Yalcindag FN, Kahraman S, Batioglu F, et al. Occlusive retinal vasculitis in a patient with ankylosing spondylitis. Eye (Lond). 2006;20:733-735.

10. Garcia-Diaz M, Mira M, Nevado L, et al. Retinal vasculitis associated with Crohn's disease. Postgrad Med J. 1995;71: 170-172. 


\section{A. M. Braakenburg and A. Rothova}

11. Matsuo T, Yamaoka A. Retinal vasculitis revealed by fluorescein angiography in patients with inflammatory bowel disease. Jpn J Ophthalmol. 1998;42:398-400.

12. Jabs DA, Nussenblatt RB, Rosenbaum JT. Standardization of uveitis nomenclature for reporting clinical data: results of the First International Workshop. Am J Ophthalmol. 2005; 140:509-516.

13. Dougados $M$, van der Linden $S$, Juhlin $R$, et al. The European Spondylarthropathy Study Group preliminary criteria for the classification of spondylarthropathy. Arthritis Rheum. 1991;34:1218-1227.

14. Khan MA. Update on spondyloarthropathies. Ann Intern Med. 2002;136:896-907.

15. Hubscher O. HLA-B27 associated spondyloarthropathy and severe ascending aortitis. J Rheumatol. 2001;28:1466.
16. Magaro' M, Altomonte L, Mirone L, et al. Seronegative spondarthritis associated with Takayasu's arteritis. Ann Rheum Dis. 1988;47:595-597.

17. Sattar MA. Coexisting HLA-B27 positive spondyloarthritis and polyarteritis nodosa. Ann Rheum Dis. 1992;51: 1338-1339.

18. Lee W, Reveille JD, Davis JC, et al. Are there gender differences in severity of ankylosing spondylitis? Results from the PSOAS cohort. Ann Rheum Dis. 2007;66:633-638.

19. Tay-Kearney ML, Schwam BL, Lowder C, et al. Clinical features and associated systemic diseases of HLA-B27 uveitis. Am J Ophthalmol. 1996;121:47-56.

20. George RK, Walton RC, Whitcup SM, et al. Primary retinal vasculitis: systemic associations and diagnostic evaluation. Ophthalmology. 1996;103:384-389. 